\title{
Video Article \\ Mechanistic Insight into the Development of TNBS-Mediated Intestinal Fibrosis and Evaluating the Inhibitory Effects of Rapamycin
}

\author{
Ramkumar Mathur ${ }^{1,2,3}$, Mahabub Maraj Alam ${ }^{4}$, Xiao-Feng Zhao ${ }^{4}$, Yunfei Huang ${ }^{4}$, Xinjun Zhu ${ }^{1,2}$ \\ ${ }^{1}$ Department of Molecular and Cellular Physiology, Albany Medical College \\ ${ }^{2}$ The IBD Center, Division of Gastroenterology, Department of Medicine, Albany Medical College \\ ${ }^{3}$ Department of Geriatrics, School of Medicine and Health Science, University of North Dakota \\ ${ }^{4}$ Department of Neuroscience and Experimental Therapeutics, Albany Medical College
}

Correspondence to: Ramkumar Mathur at ramkumar.mathur@und.edu

URL: https://www.jove.com/video/60067

DOI: doi:10.3791/60067

Keywords: Immunology and Infection, Issue 151, TNBS-mediated intestinal fibrosis, crohn's disease, inflammation, rapamycin, autophagy, resident macrophage, gene expression

Date Published: 9/12/2019

Citation: Mathur, R., Alam, M.M., Zhao, X.F., Huang, Y., Zhu, X. Mechanistic Insight into the Development of TNBS-Mediated Intestinal Fibrosis and Evaluating the Inhibitory Effects of Rapamycin. J. Vis. Exp. (151), e60067, doi:10.3791/60067 (2019).

\section{Abstract}

Significant studies have been carried out to understand effective management of intestinal fibrosis. However, the lack of better knowledge of fibrosis has hindered the development of a preventative drug. Primarily, finding a suitable animal model is challenging in understanding the mechanism of Crohn's-associated intestinal fibrosis pathology. Here, we adopted an effective method where TNBS chemical exposure to mice rectums produces substantially deep ulceration and chronic inflammation, and the mice then chronically develop intestinal fibrosis. Also, we describe a technique where a rapamycin injection shows inhibitory effects on TNBS-mediated fibrosis in the mouse model. To assess the underlying mechanism of fibrosis, we methodically discuss a procedure for purifying $\mathrm{Cx} 3 \mathrm{Cr} 1+$ cells from the lamina propria of TNBS-treated and control mice. This detailed protocol will be helpful to researchers who are investigating the mechanism of fibrosis and pave the path to find a better therapeutic invention for Crohn's-associated intestinal fibrosis.

\section{Video Link}

The video component of this article can be found at https://www.jove.com/video/60067/

\section{Introduction}

Dysregulation of immune homeostasis in the gut leads to pathogenic inflammation and has been widely known to cause inflammatory bowel disease (IBD) $)^{1,2}$. Intestinal fibrosis is a chronic consequence of inflammatory bowel diseases (IBDs), such as Crohn's disease (CD) ${ }^{3}$. The irreversible pathophysiology of CD includes intestinal stricture or stenosis of fibrosis, which limits treatment options, and with no medications currently available, the only treatment is surgery. Ultimately, the development of effective therapies to counter inappropriate inflammation is much needed to study the mechanism of $\mathrm{CD}$, and this will lead us a step closer to that.

A variety of genetic mouse models are available to study IBD including IL10 KO, SAMP/Yit and adoptive CD $45^{+}$RB high cell transfer into SCID mice ${ }^{4,5,6}$. Here, we show the procedure for TNBS-mediated fibrosis in the mouse model of CD, which is comparable to the pathology of human Crohn's fibrosis. The TNBS-induced model has certain advantages. This model is technically simple; disease onset is rapid, inexpensive, and could widely be used in different animals (e.g., mouse, rat and guinea pig ${ }^{7}$ ). Co-administration of ethanol and TNBS $(2,4,6$-trinitrobenzene sulfonic acid) abruptly damages the intestinal barrier and exposes colon tissue protein to TNBS and elicit substantial immunologic responses ${ }^{8,9}$ Repeated exposure of TNBS leads to an overreactive repair process responding to inflammation and injury, and develops a fibrotic reaction in the gut. Thus, TNBS-induced fibrosis model serves to be a very compelling model to study Crohn's-associated intestinal fibrosis.

Furthermore, mononuclear phagocytes are the primary cells that arbitrate the innate immune response to pathogenesis and injury in the gut $^{10,11,12,13}$. To elucidate the cellular mechanism and to establish the role of $\mathrm{Cx} 3 \mathrm{Cr}^{+}{ }^{+}$mononuclear phagocytes in the TNBS fibrosis model, we show the procedure of purifying the mononuclear phagocytes. Analysis of $\mathrm{C} \times 3 \mathrm{Cr} 1^{+}$cells is an essential step in order to assess the inflammatory markers and determine the concomitant mechanism for intestinal fibrosis. Collectively, this detailed procedure for TNBS fibrosis will be helpful to explain the cellular mechanisms of intestinal fibrosis.

\section{Protocol}

For this manuscript, all human samples were procured according to the approved protocol by Institute Review Board (IRB) and by the Committee on Human Research at Albany Medical College. All research involving animals were strictly followed according to the approved protocol by the 
Institutional Animal Care and Use Committee at Albany Medical College as well as the National Institutes of Health Guide for the Care and Use of Laboratory Animals.

\section{Collection of human intestinal specimens}

1. Collect human tissue samples according to the institution's research committee protocols.

2. Procure intestinal tissue (ileocolonic) of a CD patient diagnosed with fibrosis and control samples from patients with no history of IBDs.

3. Use part of the intestinal tissue for immunohistology, another part of the tissue for analyzing mRNA, and a final part for protein expression of fibrotic and cytokine genes/markers.

4. For immunohistology analysis, first fix the human tissue sample in $4 \%$ paraformaldehyde for at least $48 \mathrm{~h}$ and then transfer it into a tube containing $70 \%$ ethanol for at least $16 \mathrm{~h}$. Use this fixed tissue for making a paraffin-embedded block and cut $5 \mu \mathrm{m}$ thick tissue sections by using a tissue-microtome.

5. Using a brush, gently spread out each cut section on a glass slide for staining.

6. Stain tissue section slide with trichrome stain to detect collagen deposition, and with aSMA stain to detect myofibroblasts (See section 3 for detailed information about trichrome and aSMA staining). Examine the stained sections under a light microscope.

7. Process another part of the obtained human tissue sample to make protein lysate to detect aSMA via western blot (see section 7 for detailed information about western blot analysis).

8. Obtain RNA from the final part of human tissue sample using an extraction reagent (Table of Materials) and convert mRNA into cDNA via RT-PCR.

9. Analyze fibrotic and cytokine genes by qPCR (see section 6 for detailed information about mRNA preparation).

\section{Induction of TNBS fibrosis and rapamycin treatment in mice}

1. Carry out animal research according to the animal research protocols approved by the institution.

2. Shave adult mice around the neck area in order to pre-sensitize to TNBS via dermal exposure. Soak TNBS in a cotton swab and then apply TNBS to the shaved area of the mouse neck.

NOTE: Pre-sensitization is a very important step since it improves delayed hypersensitivity response to initiate TNBS-mediated inflammation.

3. Eight days post pre-sensitization, induce colitis by intra-rectal administration once a week for six weeks. Apply four mg of TNBS (in $25 \%$ ethanol) using a $100 \mu \mathrm{L}$ enema via a $1 \mathrm{~mL}$ syringe attached to a 3.5 French polyurethane catheter and give control mice $100 \mu \mathrm{L}$ of $25 \%$ ethanol only.

1. Anesthetize mice with pentobarbital ( $25 \mathrm{mg} / \mathrm{kg}$ intraperitoneal) or expose them to $2.5 \%$ isoflurane along with $1 \mathrm{~L} / \mathrm{min}$ of oxygen during TNBS administration. Confirm anesthetization by gently pinching toes and looking for an absence of reflex.

4. Use veterinary ointment on eyes to prevent dryness while mice are under anesthesia.

5. Inject rapamycin at $2 \mathrm{mg} / \mathrm{kg} / \mathrm{day}$ or vehicle (5\% Tween-20 and $4 \%$ ethanol) every weekday for 3-6 weeks, intraperitoneal, to both control and TNBS-treated mice.

6. Use 6-week TNBS post-treated mice intestine for analyzing the extracellular assays including histology, flow cytometry, western blotting and RNA extraction. To harvest organs, euthanize mice using $\mathrm{CO}_{2}$ inhalation and confirm euthanasia with cervical dislocation.

1. To harvest organs, euthanize mice using $\mathrm{CO}_{2}$ inhalation and confirm euthanasia with cervical dislocation. After euthanasia, longitudinally open the mouse on its ventral side using surgical grade scissors and forceps. Remove the entire colon from the rectum to the terminal ilium area. Quickly transfer the colon to ice-cold 1x HBSS and wash the colon using the same buffer.

2. Measure and compare the colon lengths of the control and TNBS-treated mice. Do this step as fast as possible to avoid drying out of the colons in order to avoid cell deaths.

3. Cut the colon into small pieces and keep at $-80^{\circ} \mathrm{C}$ for storage for future purposes (RNA/western blot analysis). Use another piece of the colon for FACS analysis.

4. Be sure to cut and collect colonic tissue samples from similar regions of the colon of both control and TNBS-treated animals NOTE: A $10 \%-20 \%$ mortality is expected with TNBS inoculation although with experience, the mortality rate can be significantly reduced.

\section{Immuno-histopathologic assessment of gut fibrosis}

1. Fix human and/or mice tissues in $4 \%$ paraformaldehyde for $48 \mathrm{~h}$, and then transfer to $70 \%$ ethanol for $16 \mathrm{~h}$. NOTE: Paraformaldehyde is a neurotoxic chemical so avoid inhaling; use a face mask while using it.

2. Paraffin embed the fixed colon tissues, slice to a $5 \mu \mathrm{m}$ thickness, and directly put the tissues on glass slides for histology

3. Perform H\&E and Trichrome Blue staining according to manufacturer's instructions to detect collagen.

1. Briefly, first deparaffinize sections by submerging the slide containing sections in two chambers of xylene for 5 min in each chamber.

2. Rinse slides with $100 \%$ alcohol for $1 \mathrm{~min}$; repeat this step three times. Rinse again with tap water for $1 \mathrm{~min}$.

3. After that, immerse slides in preheated Bouin's solution at $56{ }^{\circ} \mathrm{C}$ for $60 \mathrm{~min}$. Bouin's solution is yellow in appearance; wash after taking out the slides from Bouin's solution in tap water for $3 \mathrm{~min}$ or until slides became colorless.

4. Immerse slides in Modified Mayer's Hematoxylin solution for $7 \mathrm{~min}$ at room temperature.

5. Stain slides by immersing in Trichrome stain for 5-8 $\mathrm{min}$. Immediately, rinse slides in running water for $5-10 \mathrm{~s}$ to remove excess Trichrome stain.

6. Dehydrate slides with $100 \%$ alcohol for $1 \mathrm{~min}$. Repeat this procedure three times.

7. Clear slides with xylene for $1 \mathrm{~min}$ and repeat the step $3 x$.

8. Mount slides with mounting media (Table of Materials). 
4. Carefully hold coverslip at one end with forceps and let it cover the entire section without having any bubbles. If there are some bubbles, quickly remove bubbles by tapping the coverslip.

5. Use immunostaining to detect aSMA.

1. Block colon sections in blocking buffer $(0.2 \%$ Triton $\mathrm{X}-100$ and $5 \%$ normal goat serum in $1 \mathrm{x}$ PBS) for $1 \mathrm{~h}$ at room temperature.

2. Incubate with $100 \mu \mathrm{L}$ of diluted primary antibody for aSMA (Table of Materials) on the slide solution $(0.2 \%$ Triton $\mathrm{X}-100$ and $3 \%$ normal goat serum in 1x PBS; anti-aSMA 1:200) at $4{ }^{\circ} \mathrm{C}$ overnight.

3. Wash the sections three times with $1 \times$ PBS.

4. Use goat anti-mouse $\lg \mathrm{G}(\mathrm{H}+\mathrm{L})$ conjugated with Alexa Fluor 488 (Table of Materials) as a secondary antibody for $2 \mathrm{~h}$ at room temperature.

5. Wash the sections three times with $1 \times$ PBS.

6. Use DAPI to counterstain the nucleus for $5 \mathrm{~min}$

7. Wash the sections three times with $1 \times$ PBS.

8. Mount slides with fluorescent mounting media (Table of Materials), and seal with a coverslip using nail polish.

6. Acquire images by using LSM 880 confocal microscope and process images using microscope software.

7. Quantify images by taking an average of multiple selected areas in the same section with ImageJ.

\section{Isolation of intestinal lamina propria and purification of $\mathrm{C} \times 3 \mathrm{Cr}^{+}{ }^{+}$mononuclear phagocytes}

1. Longitudinally open the colon in ice-cold Hank's Balanced Salt Solution (HBSS; Table of Materials) and wash the colon with the same buffer.

2. Cut approximately $5 \mathrm{~cm}$ small pieces of colon tissues using sterile scissors in HBSS.

3. Transfer small colon tissue pieces in a $50 \mathrm{~mL}$ conical tube containing $10 \mathrm{~mL}$ of pre-digestion buffer (1x HBSS with $5 \%$ FBS, $5 \mathrm{mM} \mathrm{EDTA}$ and $1 \mathrm{mM}$ DTT), and shake for $20 \mathrm{~min}$ at $100 \mathrm{rpm}$ in a $37^{\circ} \mathrm{C}$ incubator ${ }^{11}$.

NOTE: Incubation of colonic tissue in $37^{\circ} \mathrm{C}$ at $100 \mathrm{rpm}$ shaking condition allows for efficient collagenase tissue digestion and high yield of viable cells.

4. Discard detached colonic epithelium by passing through a $40 \mu \mathrm{m}$ cell strainer.

5. Collect the remaining tissue from the strainer and further digest them in digestion buffer containing Collagenase type IV and DNase I (Table of Materials) in $1 \mathrm{x} \mathrm{HBSS}$ with $5 \% \mathrm{FBS}$ for $20 \mathrm{~min}$ at $37^{\circ} \mathrm{C}$ at $100 \mathrm{rpm}$.

6. Vortex the digested tissues for approximately $20 \mathrm{~s}$ and pass through a $40 \mu \mathrm{m}$ cell strainer to obtain lamina propria fractions.

7. Centrifuge the lamina propria fractions to pellet down the cells at $700 \times g$ in $4{ }^{\circ} \mathrm{C}$

8. To exclude dead cells from the lamina propria use a density gradient (Table of Materials).

NOTE: The density gradient media used here (Table of Materials) may cause loss of mononuclear cells; however, it greatly removes dead cells from the preparation, which overall increases the purity.

1. Make $100 \mathrm{~mL}$ each of $30 \%$ and $70 \%$ density gradient media solutions. Resuspend the obtained cells in $10 \mathrm{~mL}$ of the $30 \%$ solution and overlay on top of $5 \mathrm{~mL}$ of the $70 \%$ solution in a $15 \mathrm{~mL}$ tube.

2. Centrifuge the gradient in a brake-free condition at $1,000 \times g$ and room temperature. Collect the white ring phase containing lamina propria lymphocytes, which will be between the $30 \%$ and $70 \%$ gradient layers.

9. Wash the obtained cells by re-suspending in ice-cold HBSS and centrifuge at $500 \times g, 20^{\circ} \mathrm{C}$, for $10 \mathrm{~min}$. Re-suspend cells in FACS (fluorescence-activated cell sorting) buffer (PBS, pH 7.4, with $1 \%$ BSA and $2 \mathrm{mM}$ EDTA).

10. Purify mononuclear phagocytes from the collected cells purification using magnetic beads and/or FACS sorting.

1. Magnetic purification

1. Prior to staining with antibodies, first block cell surface of lamina propria cells by incubating with anti-mouse CD16/CD32 Fc blocker for $15 \mathrm{~min}$ on ice.

2. Incubate cells with anti-Cx3Cr1-PE (phycoerythrin) antibodies along with anti-PE microbeads (Table of Materials) for 30 min on ice, to capture the bound cells. Wash cells with FACS buffer.

3. Pass the antibody/bead bound cells through a magnetic-activated cell-sorting column in a magnetic field to remove unbound cells. Wash three times with FACS buffer. Remove the column from the magnetic field and push plunger in the column to yield bead bound cells.

2. FACS sorting

NOTE: FACS sorting can be used in lieu of magnetic purification. Even though magnetic purification is an easy and cost-effective method, it is limited to only purifying single cells, not for subpopulations of cells. Therefore, to isolate subpopulations of cells, the FACS sorting method is an effective method of sorting single cells as well as subpopulations of cells.

1. Block lamina propria cells with anti-mouse CD16/CD32 Fc blocker (Table of Materials).

2. Stain cells by incubating with anti-CD64, CD11c, CD11b, Cx3Cr1, Ly6C, and MHCIl antibodies.

3. Sort using a FACS flow cytometer, gating for $\mathrm{CD} 11 \mathrm{c}-\mathrm{CD} 64^{+} \mathrm{CD} 11 \mathrm{~b}^{+} \mathrm{C} \times 3 \mathrm{Cr} 1^{+} \mathrm{Ly}_{6 \mathrm{C}^{-}}$cells to purify the $\mathrm{CX} 3 \mathrm{Cr} 1(\mathrm{P} 3+\mathrm{P} 4)$ population.

11. Lyse sorted cells for total RNA preparation and detect cytokine and fibrotic markers (see section 6 for RNA and cDNA preparation).

12. Analyze mRNA expression of fibrotic markers and inflammatory cytokine analysis from isolated single-cell suspensions from magnetic purification.

13. For FACS analysis, block cells with anti-mouse CD16/CD32 Fc blocker (Table of Materials) prior to staining with antibodies against surface or intracellular markers. 


\section{Flow cytometry analysis}

1. Cell-surface staining and analysis

1. Resuspend $5-10 \times 10^{5}$ isolated lamina propria cells in $50 \mathrm{~mL}$ of FACS buffer (1\% BSA, 2 mM EDTA in PBS, pH 7.4).

2. Incubate cells with anti-mouse CD16/CD32 (Table of Materials) at a 1:50 dilution to block Fc receptors on ice for $10 \mathrm{~min}$.

3. Wash cells with $500 \mu \mathrm{L}$ of ice-cold FACS buffer to remove unbound anti-CD16/CD32 prior to cell-surface staining.

4. Surface stain colonic single-cell suspensions $\left(10^{6} \mathrm{cells} / 50 \mathrm{~mL}\right)$ with fluorescent-labelled antibody at 1:100 dilution on ice for $30 \mathrm{~min}$ to evaluate colonic lamina propria.

5. Wash labeled cells with $500 \mu \mathrm{L}$ of ice-cold FACS buffer twice to remove unbound antibodies.

6. Analyze labeled mononuclear cells by flow cytometry. Gate for Live, then for FSC ${ }^{+} S S C^{+}$followed by $\mathrm{CD} 11 \mathrm{~b}^{+} \mathrm{C} \times 3 \mathrm{Cr} 1^{+}$, and then analyze other macrophage activation markers including MHCII, CD80, CD86 and CD40.

2. Intracellular cytokine staining and analysis

1. For intracellular cytokine staining, fix and permeabilize the surface-stained cells using a Fixation/Permeabilization Solution Kit (Table of Materials); please follow the manufacturer's instructions for detailed steps.

2. Gate lamina propria cells for Live, then for $\mathrm{FSC}^{+} \mathrm{SSC}^{+}$followed by $\mathrm{CD} 11 \mathrm{~b}^{+} \mathrm{C} \times 3 \mathrm{Cr} 1^{+} \mathrm{IL}_{2} 3^{+}$to detect intracellular level of IL23 cytokine and $\mathrm{CD} 11 \mathrm{~b}^{+} \mathrm{C} \times 3 \mathrm{Cr} 1^{+} \mathrm{IL} 1 \beta^{+}$to detect intracellular level of IL1 $\beta$ cytokine.

3. aSMA staining and analysis

1. Wash cells with FACS buffer twice by centrifuging at $200 \times g$ and $4{ }^{\circ} \mathrm{C}$ for 5 min to remove excess fixative buffer.

2. To determine the aSMA level, permeabilize cells with a Fixation/Permeabilization Solution Kit (Table of Materials).

3. Incubate cells with anti-aSMA-AF488 antibody (Table of Materials) using 1:1,000 dilution on ice for $30 \mathrm{~min}$.

4. Wash cells twice and then do FACS analysis. Gate for Live, $\mathrm{FSC}^{+} \mathrm{SSC}^{+}$followed by detection of aSMA ${ }^{+}$cells in colonic cells.

\section{RNA isolation, RT-PCR, real-time PCR}

1. Isolate RNA from MACS or FACS purified cells or colon excised tissue by homogenizing them in extraction reagent (Table of Materials) NOTE: Use safety goggles and other lab protective gear when working with this reagent as it is a lung and skin irritant. Work safely in a hood.

2. Add $0.5 \mu \mathrm{L}$ of RNase-free glycogen from $20 \mu \mathrm{g} / \mathrm{mL}$ glycogen stock solution (Table of Materials) to improve the recovery of total RNA prior to precipitating RNA with isopropanol.

3. Resuspend the RNA pellet in $50 \mu \mathrm{L}$ of RNase free water (Table of Materials) and incubate at $55^{\circ} \mathrm{C}$ for 5 min to dissolve the RNA palate completely.

4. Read RNA concentrations using a spectrophotometer at $260 \mathrm{~nm}$.

5. Synthesize cDNA using $1 \mu \mathrm{g}$ of total RNA and a reverse transcription synthesis kit (Table of Materials).

6. Analyze gene expression using $2 \mu \mathrm{L}$ of cDNA as templates via real-time PCR. Use a 96-well PCR plate qPCR Master Mix kit (Table of Materials). Use CT values to calculate the fold change of RNA abundance after normalizing the GAPDH/HPRT values.

\section{Western blotting}

1. Lyse colon tissue by using RIPA lysis buffer (1\% NP40).

2. Resolve protein lysate in an $8 \%$ bis-Tris gel at a constant voltage of $80 \mathrm{~V}$.

3. Transfer gel-protein to nitrocellulose membrane(s) in cold transfer buffer running at a constant current of $50 \mathrm{~mA}$ for $2 \mathrm{~h}$ at $4{ }^{\circ} \mathrm{C}$.

4. Block non-specific regions on the protein transferred membrane using $5 \%$ non-fat milk in TBST $(25 \mathrm{mM} \mathrm{Tris-} \mathrm{HCl}, \mathrm{pH} 7.4,1.5 \mathrm{M} \mathrm{NaCl}, 0.05 \%$ Tween-20) for at least $1 \mathrm{~h}$ at room temperature.

5. To detect aSMA levels, incubate membrane(s) with aSMA primary detection antibody at $4{ }^{\circ} \mathrm{C}$ overnight.

6. Wash membranes 3-4 times using TBST in 15 min intervals.

7. Incubate with HRP-conjugated secondary antibody $(1: 10,000)$ in $5 \%$ non-fat milk in TBST.

8. Develop membranes using an ECL developing kit.

9. Normalize protein signal intensities with GAPDH as a loading control for densitometry analysis.

\section{Statistical analysis}

1. Analyze the data using data analysis software of choice by comparing between wild type and $\mathrm{KO}$ animals.

2. Consider $P$-value of $<0.05$ to be significant $\left({ }^{*} P<0.05\right.$; ${ }^{* *} P<0.01$; $\left.{ }^{* * *} P<0.001\right)$.

3. Use Student's t-test to test the differences between two groups and ANOVA test for analysis between more than two groups. 


\section{Representative Results}

We adopted the TNBS colitis mouse model to study and elucidate the underlying mechanisms of intestinal fibrosis ${ }^{8}$. Here, we performed a detailed time course study of TNBS-mediated colitis, where TNBS was rectally administrated weekly to wild type mice for up to six weeks as represented schematically (Figure 1A). After six weeks of TNBS treatment, we noticed that colonic lengths shorten progressively over the course of the TNBS treatment, from an average of $5 \pm 0.5 \mathrm{~cm}$ in the control group to $3 \pm 0.5 \mathrm{~cm}$ in the TNBS group; such quantitative analysis of colon length represents a very apparent reduction in colon length (Figure 1B). To ensure that the TNBS Crohn's disease model is comparable to the human Crohn's fibrosis model and was not an artifact related to the methodology, we analyzed the fibrotic markers at multiple levels in a detailed time course study for the TNBS injection to the wild type. Accumulation of alpha-smooth muscle actin ( $\alpha$ SMA) positive cells and collagen deposition within submucosal layers have been reported in most of the fibrosis incidences and is regarded as a hallmark for fibrotic events ${ }^{14}$. We found that the colon sections of TNBS-treated mice that were stained with aSMA showed a 4-6-fold increase in colonic submucosa layer positively stained with aSMA (Figure 1C). Besides, the Trichrome blue staining for these sections also showed a 2-4-fold increase, suggesting significant collagen deposition, which validates severe intestinal fibrosis (Figure 1D). We further assessed the activation of myofibroblasts by detecting aSMA-positive cells by FACS analysis in TNBS-treated mice colon and found a significant accumulation of aSMA-positive staining (Figure 1E). Furthermore, we found substantial induction in the expression of aSMA, Col-I, and Col-III measured by qPCR analysis in TNBStreated mice (Figure 1F). Increased expression of aSMA protein in western blot analysis revealed increased fibrosis (Figure 1G). Overall, TNBS kinetics treatment provides an opportunity to access putative immune response in chronic conditions, which closely mimics the CD chronic phase condition and is essential to fibrosis development.

To compare TNBS fibrosis with Crohn's associated fibrosis, we analyzed the expression of fibrosis markers and cytokines in fresh tissue biopsy from the ileum of patients with active CD or under remission. Remarkably, we found marked induction of thickening of aSMA-positive layers and increased collagen deposition as detected by trichrome staining in active CD sections (Figure 2A). We also performed Western blot analysis and confirmed the induction of aSMA expression in active CD samples (Figure 2B). In addition, we observed significant induction of fibrosis markers including aSMA, Col1, as detected by qPCR analysis (Figure $\mathbf{2 C}$ ).

Next, to determine a mechanism to limit TNBS fibrosis we evaluated the effects of rapamycin, a pharmacological inhibitor of mTOR activity ${ }^{15,16}$. Accordingly, we treated mice with both TNBS and rapamycin and analyzed aSMA and collagen level in colon histology and have shown quantitative measurement of aSMA and collagen in densitometry plot (Figure 3A). Our data suggests that rapamycin reduces the aSMA-positive staining in submucosal layer and lessens the collagen deposition. To further validate and quantify fibrotic responses, we determined the aSMA, collagen and TGF $\beta$ expressions by qPCR, and $\alpha$ SMA expression by flow cytometry in TNBS-treated colon (Figure 3B, 3C). We have also shown $\mathrm{C} \times 3 \mathrm{Cr}^{+}$mononuclear phagocytes induce an inflammatory immune response to injury ${ }^{9}$. Thus, we wanted to see if administration of rapamycin in TNBS-treated mice could reverse the inflammatory and fibrotic effects. Therefore, we purified $\mathrm{Cx} 3 \mathrm{Cr} 1^{+}$resident mononuclear phagocytes from colonic single cell suspensions by using magnetic microbeads (Figure 3D). We found an increased level of $p$-p70 and p-S6 in Cx3Cr $1^{+}$resident mononuclear phagocytes by western blot analysis from TNBS-treated mice, and this level was blocked by rapamycin (Figure 3E). Besides, we found that rapamycin treatment dampens down the IL-23 and IL-1 $\beta$ in the TNBS-treated group (Figure 3F). Moreover, these findings elucidate the effective mechanism involved in the induction of TNBS-fibrosis and have shown that rapamycin attenuates the induction of fibrosis.

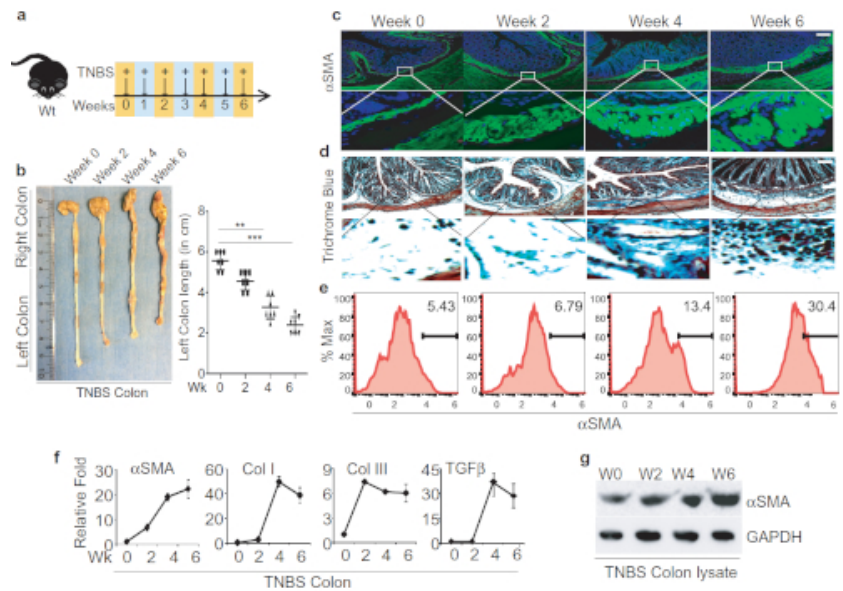

Figure 1: Successful TNBS administration leads to developing intestinal fibrosis in mice. A. Schematic diagram representation of weekly TNBS treatment given to wild-type mice. B. Colon images and measurement of colon lengths from TNBS-treated mice, harvested on weeks 0 , 2,4 , and 6 post-TNSB treatment. C-D. Colon sections' histological analysis stained with anti-aSMA antibody for activation of myofibroblasts and trichrome blue staining for collagen; scale bar $100 \mu \mathrm{m}$. E. FACS analysis to identify aSMA-positive cells in the colon and quantification of aSMApositive cells. F. Fibrotic markers and cytokines detected by qPCR. G. western blot analysis of aSMA from colon lysate of TNBS-treated and control mice. This modified figure is being reused with the permission of previous publication ${ }^{9}$ in Mucosal Immunology, 2019 by Mathur et al. Please click here to view a larger version of this figure. 


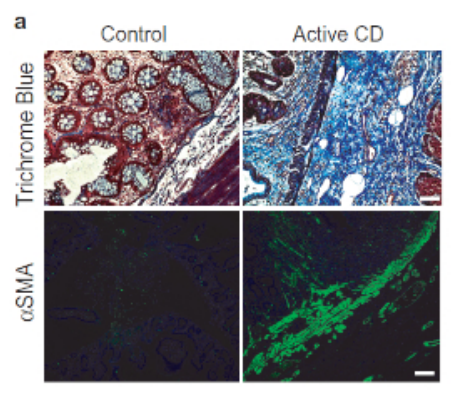

Human Colon

b

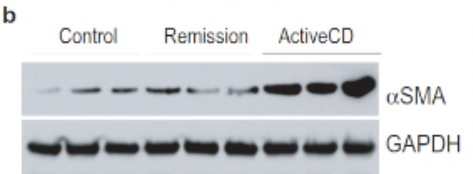

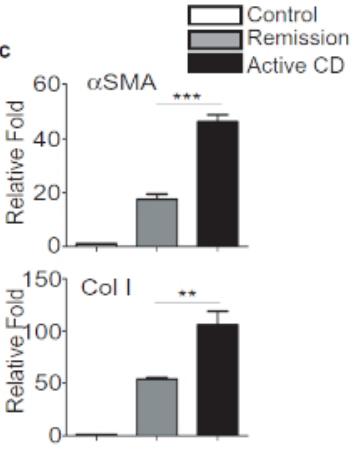

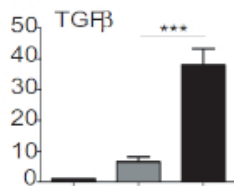

Figure 2: TNBS Fibrosis is comparable to Crohn's-associated Intestinal fibrosis. A. Representative images of colon biopsies of control, active CD showing a significant increase of trichrome blue staining and aSMA-positive staining in submucosal layers, scale bar $50 \mu \mathrm{m}$. B. Western blot analysis of aSMA expression and quantification. C. qPCR analysis of fibrotic markers and cytokines. This modified figure is being reused with the permission of previous publication ${ }^{9}$ in Mucosal Immunology, 2019 by Mathur et al. Please click here to view a larger version of this figure.

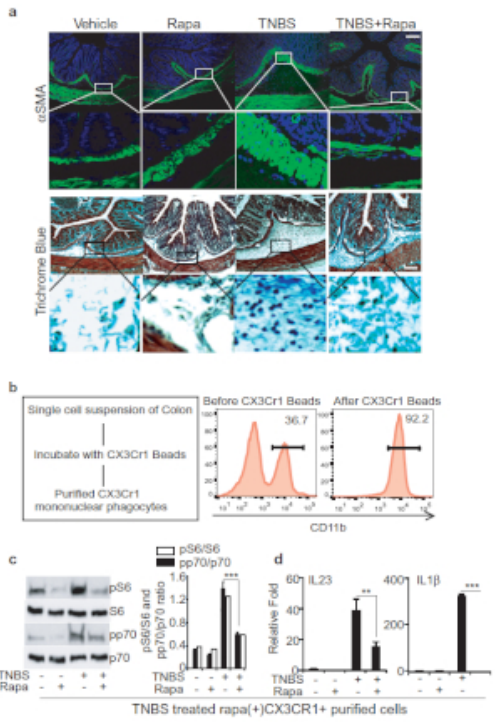

Figure 3: Rapamycin treatment effectively ameliorates TNBS-induced fibrosis. A. Colon histological analysis - representative images of myofibroblast staining with anti-aSMA antibody and collagen staining with Trichrome blue, scale bar $100 \mu \mathrm{m}$. B. qPCR analysis of $\alpha \mathrm{SMA}$, Collagen and TGF $\beta$ expression in Control/TNBS-treated mouse colons. C. FACS analysis of aSMA in single cell suspension from mouse colon treated with Control/TNBS. D. Schematic for purification of $\mathrm{C} \times 3 \mathrm{Cr}^{+}{ }^{+}$cells from colonic lamina propria fraction and FACS analysis of purified cells. E. Western blot analysis of p-p70 and p-S6 levels in purified $\mathrm{C} \times 3 \mathrm{Cr} 1+$ mononuclear phagocytes from mice treated with TNBS and/or rapamycin. F. qPCR analysis of the expression in purified Cx3Cr1+ mononuclear phagocytes, which produces IL-23 and IL-1 $\beta$. This modified figure is being reused with the permission of previous publication ${ }^{9}$ in Mucosal Immunology, 2019 by Mathur et al. Please click here to view a larger version of this figure.

\section{Discussion}

Wound healing or tissue repair is a tightly regulated biological process ${ }^{17}$. During tissue injury with a chemical, mechanical and infection condition, an inflammatory response triggers the tissue repair process. However, a dysregulated and pathological inflammatory response leads to developing scarring or a fibrotic reaction, which could impair the tissue repair function ${ }^{9,18,19}$. Here, we show the procedure for the TNBS-induced fibrosis animal model, which significantly shares pathophysiology with human Crohn's disease. Successive inoculation of TNBS chemical exposure to damage the mouse epithelium causes deep ulcerations and induces fibrosis development. With a reliable, low cost, and rapid induction of disease onset, this method is widely accepted by multiple research groups involved in studies for tissue injury, transmural inflammation and the gut-brain axis.

To successfully implement the TNBS fibrosis model, there are several essential steps. For example, adequate dosage and timing of TNBS administration are very important. A 6-8-week TNBS inoculation allows deep tissue ulceration and is highly recommended for chronic fibrotic studies. Outcoming stool from mice and the retrograde reflex of inoculated TNBS are two major problems, which could result in delivery of 
variable doses to mice. Gentle pressure near the mice rectum may help release stools before TNBS administration. Holding the head of the animal down for a few seconds dramatically helps to stop reverse reflux of TNBS. Keeping the mice in a cage, placing the cage on a heat pad, and providing the mice with Napa nectar could also be useful strategies in preventing high mortality.

Here, we also discuss the gut inflammation during TNBS fibrosis and their impact on fibrotic response. mTOR/autophagy role is broadly implicated on intestinal homeostasis and in IBD pathogenesis ${ }^{20,21}$. We identified that mTOR/autophagy signaling is critical to modulate proinflammatory responses from IL-23 and IL $1 \beta$ cytokines from $\mathrm{Cx} 3 \mathrm{Cr}^{+}{ }^{+}$mononuclear phagocytes that affect pro-fibrotic IL-23/IL-22 axis (Figure $3 \mathrm{~A})^{9}$. Thereby, purifying single $\mathrm{Cx} 3 \mathrm{Cr}^{+}{ }^{+}$mononuclear cells for immunoprofiling and gene expression is a critical step of the model. We discuss in detail the protocol for isolating lamina propria and provide the purification procedure for $\mathrm{C} 3 \mathrm{Cr}^{+}$mononuclear cells using magnetic beads.

Collectively, despite the presence of genetic and spontaneous models for Crohn's disease, TNBS-colitis remains a potent tool to study the immuno-pathogenesis of CD and has the potential to evaluate the Crohn's fibrosis treatments. The major limitation of using chemically induced fibrosis models such as TNBS is the chance of high variability from user to user and the possibility of outliers in the data. A sample size of 5-8 animals per group along with greater user experience can increase the consistency of the model. However, finding a suitable genetic model causing spontaneous Crohn's fibrosis is and will always be warranted.

\section{Disclosures}

Competing interests: The authors declare no competing interests.

\section{Acknowledgments}

This work was supported by NIH grant R01NS093045 (Y.H.), NIH grant K08DK088950 (X.Z.), R03DK099566 (X.Z.), and The Crohn's \& Colitis Foundation of America research Fellowship (CCFA) 481637 (R.M.).

\section{References}

1. Garrett, W. S., Gordon, J. I., Glimcher, L. H. Homeostasis and inflammation in the intestine. Cell. 140, 859-870 (2010).

2. Park, S. G. et al. T regulatory cells maintain intestinal homeostasis by suppressing gammadelta T cells. Immunity. 33, 791-803 (2010).

3. Speca, S., Giusti, I., Rieder, F., Latella, G. Cellular and molecular mechanisms of intestinal fibrosis. World Journal of Gastroenterology. 18, 3635-3661 (2012).

4. Keubler, L. M., Buettner, M., Hager, C., Bleich, A. A Multihit Model: Colitis Lessons from the Interleukin-10-deficient Mouse. Inflammatory Bowel Disease. 21, 1967-1975 (2015).

5. Strober, W., Nakamura, K., Kitani, A. The SAMP1/Yit mouse: another step closer to modeling human inflammatory bowel disease. Journal of Clinical investigation. 107, 667-670 (2001).

6. Ostanin, D. V. et al. T cell transfer model of chronic colitis: concepts, considerations, and tricks of the trade. American Journal of PhysiologyGastroenterology and Liver Physiology. 296, G135-146 (2009).

7. Antoniou, E. et al. The TNBS-induced colitis animal model: An overview. Annals of Medicine and Surgery. 11, 9-15 (2016).

8. Loeuillard, E. et al. 2,4,6-trinitrobenzene sulfonic acid-induced chronic colitis with fibrosis and modulation of TGF-beta1 signaling. World Journal of Gastroenterology. 20, 18207-18215 (2014).

9. Mathur, R. et al. Induction of autophagy in Cx3cr1(+) mononuclear cells limits IL-23/LL-22 axis-mediated intestinal fibrosis. Mucosal Immunology. 12, 612-623 (2019).

10. Joeris, T., Muller-Luda, K., Agace, W. W., Mowat, A. M. Diversity and functions of intestinal mononuclear phagocytes. Mucosal Immunology. 10, 845-864 (2017).

11. Mathur, R. et al. A mouse model of Salmonella typhi infection. Cell. 151, 590-602 (2012).

12. Zhao, X. et al. Noninflammatory Changes of Microglia Are Sufficient to Cause Epilepsy. Cell Reports. 22, 2080-2093 (2018).

13. Murugaiyan, G. et al. MicroRNA-21 promotes Th17 differentiation and mediates experimental autoimmune encephalomyelitis. Journal of Clinical investigation. 125, 1069-1080 (2015).

14. Hinz, B., Celetta, G., Tomasek, J. J., Gabbiani, G., Chaponnier, C. Alpha-smooth muscle actin expression upregulates fibroblast contractile activity. Molecular Biology of the Cell. 12, 2730-2741 (2001).

15. Yang, J. et al. Rapamycin Inhibition of mTOR Reduces Levels of the Na+/H+ Exchanger 3 in Intestines of Mice and Humans, Leading to Diarrhea. Gastroenterology. 149, 151-162 (2015).

16. Mutalib, M. et al. The use of sirolimus (rapamycin) in the management of refractory inflammatory bowel disease in children. Journal of Crohns and Colitis. 8, 1730-1734 (2014).

17. Shaw, T. J., Martin, P. Wound repair at a glance. Journal of Cell Science. 122, 3209-3213 (2009).

18. Paul, J. et al. IL-17-driven intestinal fibrosis is inhibited by Itch-mediated ubiquitination of HIC-5. Mucosal Immunology. 11, 427-436 (2018).

19. Sohail, I., Ghosh, S., Mukundan, S., Zelewski, S., Khan, M. N. Role of Inflammatory Risk Factors in the Pathogenesis of Streptococcus pneumoniae. Frontiers of Immunology. 9, 2275 (2018).

20. Laplante, M., Sabatini, D. M. mTOR signaling in growth control and disease. Cell. 149, 274-293 (2012).

21. Xavier, R. J., Podolsky, D. K. Unravelling the pathogenesis of inflammatory bowel disease. Nature. 448, 427-434 (2007). 